\title{
Clinical improvement of vasovagal syncope in the absence of specific therapies: The Seinfeld effect*
}

\author{
Inderjeet Sahota, Robert Sheldon, Payam Pournazari \\ Libin Cardiovascular Institute of Alberta, University of Calgary, Calgary, Canada
}

\begin{abstract}
Vasovagal syncope can persist for decades and recur sporadically but many patients appear to improve after being seen in specialty clinics. The absence of specific and proven effective therapy raises the possibility that this might be due to regression to the mean or to a placebo effect. However, analysis using the Poisson distribution indicates the extreme unlikeliness that regression to the mean is the explanation. A main cause of the placebo effect is expectancy. Subject expectancy is the influence of the subject's anticipation of benefit on outcomes, and observer expectancy is the influence of investigator or physician attitudes and behavior on subject response. Ample data support the role of expectancy in outcomes of syncope patients. Moreover, expectancy can vary depending on the type of ineffective intervention. Interestingly, studies in which patients are blinded but the investigator is not show similar patient benefits compared with completely open label studies consistent with a strong observer expectancy effect due to physician-subject interaction. These results suggest the paramount importance of properly conducted randomized clinical trials in assessing biomedical interventions, and also illuminate the powerful potential of studies aimed at enhancing the expectancy effect on patient outcome. (Cardiol J 2014; 21, 6: 637-642)
\end{abstract}

Key words: vasovagal syncope, placebo, Poisson, natural history, expectancy

\section{The clinical problem}

One of the great challenges in assessing and treating vasovagal syncope (VVS) is its sporadic nature. Numerous discussions suggest susceptibility of this patient population to the placebo effect. We believe the conversation needs to be recast. Placebo generally involves an implicit or explicit element of deliberate deception by physicians; this distracts investigators from seeing the opportunities of the real issue: the expectancy effect.

At least $40 \%$ of people have a vasovagal faint by age 60 , and in most fainters, it is a recurring problem $[1,2]$. In young and middle-aged community populations, the median lifetime number of faints approximates $3-4$, but clinical populations in observational and randomized trials often report a median of 10-20 lifetime faints. Only a minority faint once. Therefore, VVS is common, and commonly recurrent.

What is particularly interesting and germane is variation of syncope frequency within any single patient. For nearly 20 years, observational and interventional studies have noted qualitatively that patients appear to fare better after being seen in specialty clinics than might be expected from their clinical history. This was first noticed in a series of retrospective observational studies from our center [3-5]; since then, this has been seen ubiquitously in single center and multicenter studies. It has led to randomized controlled trials being much larger than might have been predicted based on baseline

\footnotetext{
Address for correspondence: Robert Sheldon, MD, PhD, University of Calgary, 3280 Hospital Drive NW, Calgary, Alberta T2N 4N1, Canada, e-mail: sheldon@ucalgary.ca Received: 9.10.2014 Accepted: 13.10.2014

*A show about nothing (http://en.wikipedia.org/wiki/Seinfeld).
} 
symptom burden in order to provide adequate power. The common conclusion is that a powerful placebo effect is at play, but is this the correct, or only, possibility?

Here, we review some common explanations for temporal variability in the clinical course of syndromes with paroxysmal presentations, then try to draw tentative conclusions about the nature of temporal variability in VVS.

\section{The nature of clinical variance}

\section{Regression to the mean}

Recurring events can be described with mathematical modeling. Theoretically, some paroxysmal events recur with a pattern of true temporal randomness, with independence of 1 event and another. Commonly, these events fit a Poisson distribution, which can be depicted by the classic survival curve well known in the medical literature. A related and common variant is the Weibull distribution. Both assume a random series of events from the population of patients; that is, the random recurrence of syncope over time.

In random models, some events occur earlier and some later, leading to the appearance of long and short intervals between events, and periods of more and less frequent fainting. An observer might conclude that the syndrome is entering a quiescent phase when going from times of frequent to less frequent events. In truth, the apparent improvement in syncope frequency might simply be regression to the mean.

\section{Placebo effect}

The true placebo effect is a complex and comprehensive collection of factors [6-9]. Although the term is used widely, there is legitimate debate as to whether administering a purposefully deceptive intervention - the placebo proper - has any effect above non-biomedical but behavioral interventions and effects such as conditioning [6], expectancy [9], and physician behavior $[7,8,10]$. Kirsch originally hypothesized [9] that placebo effects are produced by a self-fulfilling effect. If a person believes that he or she will feel different, they actually feel different. This is termed "response expectancy". Subjects who expect to feel better demonstrate subject response expectancy, while subjects whose physicians expect them to feel better demonstrate observer response expectancy.

Placebo effects can be observed in invasive and non-invasive studies, although there is debate whether a placebo intervention is better than no intervention at all $[7,8,10]$. Arthroscopic partial meniscectomy is frequently used to reduce symptoms due to a tear in a meniscus. The procedure is very common, with a widespread understanding that it is effective at reducing symptoms. However, Sihvonen et al. [11] performed a randomized controlled study of arthroscopic meniscectomy comparing "real" to sham surgery. Personnel outside the operating room did not know the intervention allocation until completion of data analysis. At 12 months, no significant difference in symptoms existed between groups suggesting that a major aspect of symptom suppression was due to expectancy on the part of patients and surgeons. A clinical trial of back surgery for osteoporotic fractures came to the same conclusion [12].

The same effect occurs in hypertrophic obstructive cardiomyopathy patients undergoing pacemaker implantation. Initial reports concluded that permanent cardiac pacing reduced symptoms in these patients, but, in a double-blind randomized trial, Linde et al. [13] found no differences in chest pain, dyspnea or dizziness between active and inactive pacing. The placebo (and likely expectancy) effect is, therefore, a real response, that plays an important role in "treatment".

Not everyone is susceptible to this type of placebo effect [14] raising the possibility of a genetic basis to the placebo effect, in general, and to syncope, in particular. Furmark et al. [15] reported that the magnitude of the placebo response in patients with social anxiety disorder is linked to attenuation in amygdala excitability, which, in turn, is linked to specific alleles of the serotonin transporter and tyrosine hydroxylase. This mechanism may be of particular importance to syncope given the strongly suspected relationship between serotonin signaling and syncope. Similarly, Hall et al. [16] reported that polymorphisms in catechol-O-methyltransferase are associated with increased responsiveness to the placebo effect. This effect is likely due to an interaction with dopamine availability in the prefrontal cortex.

\section{Variance in syncope presentation}

In randomized clinical trials of VVS, about $99 \%$ of patients faint in the year before randomization, but usually less than half faint in the next year. Numerous retrospective and prospective observational studies and randomized studies with a wide range of blinding, controls, and populations provide interesting test cases with which we can explore possible reasons for why people appear to stop fainting. 


\section{Regression to the mean}

The Poisson distribution permits estimating the distribution that characterizes events with low occurrence rates in time. Poisson originally derived it to describe gamblers' wins and losses in a game with a low likelihood of wins. Some interesting historical applications included estimating the number of false convictions in early $19^{\text {th }}$ century France, the likelihood of getting kicked by a horse in the Prussian army, and assessing whether flying bombs in World War II were targeting specific areas in Britain with either high or low rates of explosions.

The Poisson distribution can estimate the likelihood that a patient with a specific number of faints in 1 year will not faint in the next, all factors being unchanged. Most randomized clinical trials of syncope include patients who had fainted a median of 2-5 times in the preceding year, yet, in most studies, only a minority faint again the next year. For patients with $2,3,4$, and 5 faints in the prior year, the probabilities of no faints in the next year are $14 \%, 5 \%, 1.8 \%$, and $0.7 \%$. Therefore, it seems exceedingly unlikely that the apparent improvement in syncope patients is simply due to a statistical fluctuation in syncope frequency.

\section{Improvement with no placebo}

The ISSUE 1 study [17] was a prospective observational study in which all the enrolled subjects received an implantable loop recorder, and few received a specific intervention. There was a large reduction in the likelihood of subjects fainting, yet there was no placebo administered. Improvement occurred without deception or offer of clinical gain. In the PC trial, van Dijk et al. [18] reported that physical maneuvers reduced the likelihood of a syncope recurrence compared to no specific treatment. However, the control arm, whose treatment consisted generally of lifestyle and dietary suggestions of dubious biomedical value, also showed a reduction in syncope probability after study enrolment. That is, a lifestyle placebo appeared to have an effect on reducing syncope recurrences.

Similar effects are seen in open-label, randomized, pacemaker studies. For example, in the VASIS study [19], all patients fainted in the preceding year, with a median frequency of 3 faints per year, yet over an extended follow-up, fully $39 \%$ did not faint at all. Therefore, conservative treatment, without a known biological effect, provided sustained benefit. Again, a lifestyle placebo appears to have an effect on reducing syncope recurrences.

\section{Ineffective treatment \\ vs. no specific treatment}

We reported a series of observational studies [3-5] in which some patients received what was imagined and conveyed as active treatment, while others did not. This was solely on the basis of physician's clinical decision-making, and the medications subsequently were shown to be ineffective. Both groups showed a marked and similar reduction in the proportion of patients who fainted, providing evidence that administering biologically ineffective treatment (in good faith) is no better than no specific treatment. That is, a medical placebo appears to have no more powerful effect than interaction with a specialist physician [10].

\section{Effect of placebo type}

There are multiple observational studies and randomized trials that provide insight into invasive vs. non-invasive placebo. In the first Prevention of Syncope Trial [20] almost all patients fainted in the year before enrollment, with a median of 3 faints in that year, but $60 \%$ taking a medical placebo did not faint in the follow-up year. Similarly, in the double-blinded VPS II [21] and SYNPACE trials [22], the control inactive pacemaker arms had a marked reduction in syncope frequency compared to the preceding interval. Therefore, patients receiving either medical or device placebos have a marked reduction in syncope likelihood.

\section{Effect of expectancy}

These cases suggest that there is a powerful beneficial force embedded in the physician-patient relationship [23, 24], independent of whether a biomedical placebo is deliberately, even inadvertently, administered. It is also independent of whether the placebo is noninvasive or invasive. Is there a gradation within this effect?

One study compared invasive and non-invasive approaches to VVS [25]. This prospective, open-label, controlled trial assigned patients to medical (beta-blocker) or invasive (permanent cardiac pacing) "treatment". Both interventions were later shown in placebo-controlled randomized studies to have little or no true benefit. Both groups had a considerable decline in the frequency of symptoms in follow up, and the patients with pacemakers had far fewer outcomes that the patients with beta-blockers. Essentially, this was a randomized controlled trial of invasive vs. non-invasive placebo, and patients with the invasive placebo had a better outcome suggesting that treatment expectation is higher with invasive placebo. 


\section{Effect of observer expectancy}

In an informative meta-analysis, Sud et al. [26] reported that the likelihood of a syncopal recurrence was reduced in patients with active pacing vs. no implanted pacemaker; this was not significantly reduced in double-blinded randomized trials. Strikingly, in single-blinded studies in which the patients, but not physicians, were blinded to the treatment allocations, there was a large reduction in syncope likelihood. The authors concluded that the observed differences among study types were the result of physician behaviors leading to ascertainment bias or to the expectancy effect.

\section{Syncope, expectancy, and improvement}

The variation in syncope frequency provides ample opportunity for understanding and using expectancy to benefit our patients. There is relatively little evidence that a true placebo effect is at play: patients seem to improve about as much when they receive no specific treatment as when they receive inactive biomedical treatment regardless of the study type (observational vs. randomized), or the control arm. The common theme is that they are seen in specialty clinics providing a unique value of and expectations attached to the syncope "expert".

\section{Implications for understanding and treating syncope}

These studies raise several interesting questions concerning the natural history and treatment of syncope. We have shown that patients present for specialist assessment after a recent and marked worsening in syncope frequency [27], and that their syncope frequency after assessment can be predicted by their recent syncope frequency, and not syncope frequencies in the distant past [28]. We also show that syncope recurrence can improve substantially based on expectancy.

This raises the converse issue: why do people worsen? One possibility is that patients, for reasons unknown, develop negative subject expectancy, and subconsciously, anticipate or expect that they will faint again. That is, the first faint of a cluster is sporadic, then negative subject expectancy sustains the cluster, and positive observer and possibly subject expectancy reduces or eliminates syncope frequency.

Understanding syncope variation will have an impact on the structure of randomized clinical trials. It may be that patients should be screened first for susceptibility to expectancy, possibly with genetic screening. This would allow focus only on patients unlikely to respond to conservative measures, and this would have a major impact on power calculations and possibly on study feasibility. VVS requiring biomedical intervention may become a rare disease!

There may also be genetic and mechanistic implications [15, 16]. Genes with known association with the placebo response also have plausible connections to VVS. Is there an unusually strong connection between VVS and expectancy and, if so, can the course of VVS be modified by therapies targeting the expectancy effects? This will raise a host of ethical issues, given the close interactions among observer and subject expectancy and the possible use of enthusiastically positive shams to effect a successful outcome. Conversely, are there patients who will purposely fail any expectancy or drug effects? That is also conceivable.

\section{Ethics of placebo in clinical practice}

Many clinicians are unwilling to use placebos that require active concealment or deception. On the one hand, clinicians are expected to respect their patient's autonomy and informed consent whereas, on the other hand, harnessing the power of placebo may require deception. We contend that at least for VVS, the argument may be irrelevant, in that for suppressing VVS, physician-patient relationship is critical. The biomedical intervention may be needed in only a residual minority. Indeed, Margo [8] recently suggested that the physician is a placebo.

\section{Placebo in practice}

Lichtenberg et al. [29] argue that the "placebo is deception only for those who would reduce treatment to a purely biomedical pursuit”. Physicians intervene in many non-biomedical ways in caring for their patient - including assurance, personality, encouragement, offers to help and resolving uncertainty. In this light, the expectancy effect is just one in a spectrum of intangible ways a physician helps improve patient health.

Lichtenberg et al. [29] suggested when placebo use is acceptable, when the intent is to help patients and not silence them, when ineffective placebos should be withdrawn, and when they do not supplant proven therapies. Olshansky [23] argued that if the placebo effect is strong, the risk of harm is low, and other treatment options are limited, the use of placebo may be justified regardless of its deceptive underpinnings. One could argue that all 
of these criteria are met in patients with recurrent syncope, especially given how powerful the effect appears to be.

Counterarguments to placebo use also require consideration. Asai and Kadooka [30] raised several major objections. The act of prescribing a deceptive placebo prevents true informed consent. Secondly, for placebos to be at all ethical they would need to work. A meta-analysis of 114 randomized trials in which patients received either placebo or no treatment suggests that this may not be the case [10]. However, this would be irrelevant if the nominal "no-treatment" featured earnest observer expectancy. Thirdly, included in the risks of placebo administration should be the consequences of a breakdown in physician-patient trust, but again this would be irrelevant if observer expectancy is the intervention. Finally, the authors argue that deception in placebo opposes the idea of dignity and may lead to patient's unhappiness.

\section{Expectancy and practice}

The effect of expectancy is real, not due to placebo. Indeed, the term placebo frequently carries vaguely negative connotations in this syndrome. Rather, temporal variation and the expectancy effect reflect fundamental aspects of the genetics, physiology, and treatment of VVS.

This understanding should have a liberating effect on physicians' attitudes and practice. $\mathrm{Pa}-$ tients do not need to be deceived with intentional placebos; indeed, deliberate deception is probably irrelevant. Virtually nothing is taught in medical schools or postgraduate training about placebo effects and the effect of physicians' attitudes and behaviors, yet this seems critical for effective therapy in VVS. Expectancy indicates that patients respond better when physicians are optimistic and positive about recommendations. The glass should always be half-full, and not half empty. For example, we might state that the treatment works well in many or most patients (be honest), and that we expect that it will work well for the person across the desk. We, ourselves, are forces for good, in addition to whatever biomedical treatments and benefits are available.

\section{Conflict of interest: None declared}

\section{References}

1. Serletis A, Rose S, Sheldon AG, Sheldon RS. Vasovagal syncope in medical students and their first-degree relatives. Eur Heart J, 2006; 27: 1965-1970.
2. Ganzeboom KS, Mairuhu G, Reitsma JB, Linzer M, Wieling W, van Dijk N. Lifetime cumulative incidence of syncope in the general population: A study of 549 Dutch subjects aged 35-60 years. J Cardiovasc Electrophysiol, 2006; 17: 1172-1176.

3. Sheldon R, Rose S, Flanagan P, Koshman ML, Killam S. Effect of beta blockers on the time to first syncope recurrence in patients after a positive isoproterenol tilt table test. Am J Cardiol, 1996; 78: 536-539.

4. Sheldon R, Rose S, Flanagan P, Koshman ML, Killam S. Risk factors for syncope recurrence after a positive tilt-table test in patients with syncope. Circulation, 1996; 93: 973-981.

5. Sheldon R, Rose S, Koshman ML. Comparison of patients with syncope of unknown cause having negative or positive tilt-table tests. Am J Cardiol, 1997; 80: 581-585.

6. Voudouris NJ, Peck CL, Coleman G. The role of conditioning and verbal expectancy in the placebo response. Pain, 1990; 43: 121-128.

7. Miller FG, Rosenstein DL. The nature and power of the placebo effect. J Clin Epidemiol, 2006; 59: 331-335.

8. Margo CE. The placebo effect. Surv Ophthalmol, 1999; 44: 31-44.

9. Kirsch I. Response expectancy as a determinant of experience and behavior. Am Psychologist, 1985; 40: 1189-1202.

10. Hrobjartsson A, Gotzsche PC. Is the placebo powerless? An analysis of clinical trials comparing placebo with no treatment. N Engl J Med, 2001; 344: 1594-1602.

11. Sihvonen R, Paavola M, Malmivaara A et al. Arthroscopic partial meniscectomy versus sham surgery for a degenerative meniscal tear. N Engl J Med, 2013; 369: 2515-2524.

12. Kallmes DF, Comstock BA, Heagerty PJ et al. A randomized trial of vertebroplasty for osteoporotic spinal fractures. N Engl J Med, 2009; 361: 569-579.

13. Linde C, Gadler F, Kappenberger L, Ryden L. Placebo effect of pacemaker implantation in obstructive hypertrophic cardiomyopathy. PIC Study Group. Pacing In Cardiomyopathy. Am J Cardiol, 1999; 83: 903-907.

14. Levine JD, Gordon NC, Bornstein JC, Fields HL. Role of pain in placebo analgesia. Proc Natl Acad Sci USA, 1979; 76: 3528-3531.

15. Furmark T, Appel L, Henningsson S, Ahs F et al. A link between serotonin-related gene polymorphisms, amygdala activity, and placebo-induced relief from social anxiety. J Neurosci, 2008; 28: 13066-13074.

16. Hall KT, Lembo AJ, Kirsch I et al. Catechol-O-methyltransferase val158met polymorphism predicts placebo effect in irritable bowel syndrome. PLoS One, 2012; 7: e48135.

17. Moya A, Brignole M, Menozzi C et al. Mechanism of syncope in patients with isolated syncope and in patients with tilt-positive syncope. Circulation, 2001; 104: 1261-1267.

18. van Dijk N, Quartieri F, Blanc JJ et al. Effectiveness of physical counterpressure maneuvers in preventing vasovagal syncope: The Physical Counterpressure Manoeuvres Trial (PC-Trial). J Am Coll Cardiol, 2006; 48: 1652-1657.

19. Sutton R, Brignole M, Menozzi C et al. Dual-chamber pacing in the treatment of neurally mediated tilt-positive cardioinhibitory syncope : pacemaker versus no therapy: A multicenter randomized study. The Vasovagal Syncope International Study (VASIS) Investigators. Circulation, 2000; 102: 294-299.

20. Sheldon R, Connolly S, Rose S et al. Prevention of Syncope Trial (POST): A randomized, placebo-controlled study of metoprolol in the prevention of vasovagal syncope. Circulation, 2006; 113: 1164-1170. 
21. Connolly SJ, Sheldon R, Thorpe KE et al. Pacemaker therapy for prevention of syncope in patients with recurrent severe vasovagal syncope: Second Vasovagal Pacemaker Study (VPS II): A randomized trial. J Am Med Assoc, 2003; 289: 2224-2229.

22. Raviele A, Giada F, Menozzi C et al. A randomized, double-blind, placebo-controlled study of permanent cardiac pacing for the treatment of recurrent tilt-induced vasovagal syncope. The vasovagal syncope and pacing trial (SYNPACE). Eur Heart J, 2004; 25: $1741-1748$.

23. Olshansky B. Placebo and nocebo in cardiovascular health: implications for healthcare, research, and the doctor-patient relationship. J Am Coll Cardiol, 2007; 49: 415-421.

24. Thomas KB. General practice consultations: is there any point in being positive? Br Med J (Clin Res Ed), 1987; 294: 1200-1202.

25. Ammirati F, Colivicchi F, Santini M. Permanent cardiac pacing versus medical treatment for the prevention of recurrent va- sovagal syncope: A multicenter, randomized, controlled trial. Circulation, 2001; 104: 52-57.

26. Sud S, Massel D, Klein GJ et al. The expectation effect and cardiac pacing for refractory vasovagal syncope. Am J Med, 2007; 120: 54-62.

27. Sheldon RS, Sheldon AG, Serletis A et al. Worsening of symptoms before presentation with vasovagal syncope. J Cardiovasc Electrophysiol, 2007; 18: 954-959.

28. Sumner GL, Rose MS, Koshman ML, Ritchie D, Sheldon RS. Recent history of vasovagal syncope in a young, referral-based population is a stronger predictor of recurrent syncope than lifetime syncope burden. J Cardiovasc Electrophysiol, 2010; 21: 1375-1380.

29. Lichtenberg P, Heresco-Levy U, Nitzan U. The ethics of the placebo in clinical practice. J Med Ethics, 2004; 30: 551-554.

30. Asai A, Kadooka Y. Reexamination of the ethics of placebo use in clinical practice. Bioethics, 2013; 27: 186-193. 